\title{
The first 100 cases of paediatric transnasal extended endoscopic procedures in Birmingham (UK)
}

Neil Giblett ${ }^{1}$, Manoj Parulekar ${ }^{2}$, Karen Bradshaw ${ }^{2}$,

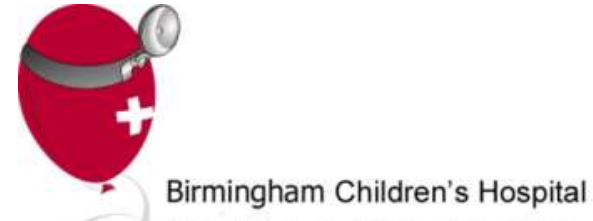
Ear, Nose and Throat Surgery
Desi Rodrigues ${ }^{2}$, Shahzada Ahmed ${ }^{3}$, Ann-Louise McDermott ${ }^{2}$

\section{Institution}

1.Specialist Trainee Registrar in Otolaryngology, West Midlands

2.Birmingham Children's Hospital

3.University Hospitals Birmingham

\section{Background:}

Extended transnasal endoscopic procedures including anterior skull-base surgery are regularly performed in adults but their use and outcomes are not well recognised in a paediatric setting.

\section{Objectives:}

To evaluate our experience of extended paediatric endoscopic sinonasal and skull-base surgery.

\section{Methods:}

We report on the first 100 cases between 2009 and 2017 of extended sinonasal and anterior skull base surgery performed at Birmingham Children's Hospital.
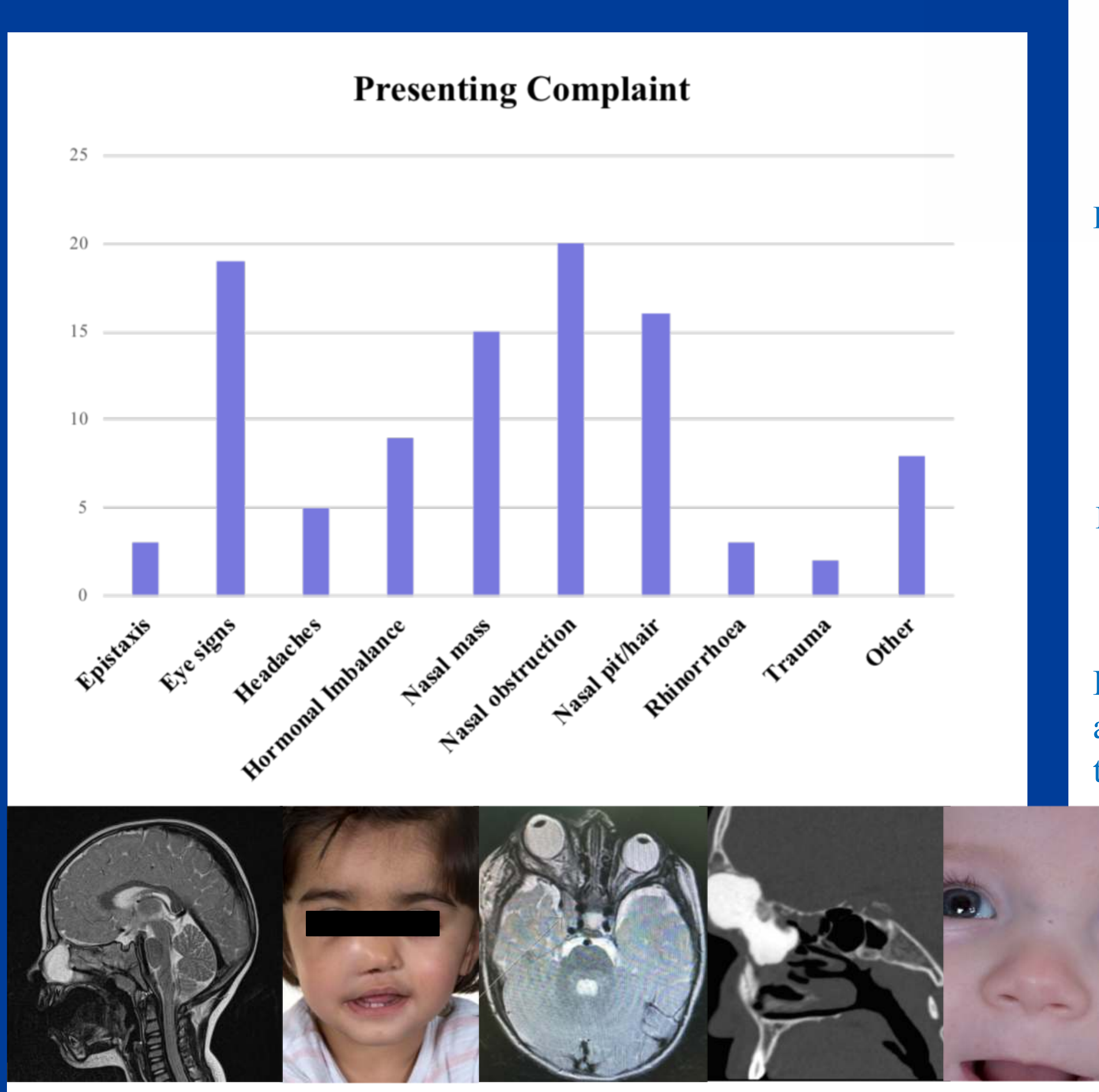

\section{Results:}

109 procedures were performed on 100 children with a mean age of 5.4 years (range; 1month-17years) and a male preponderance.

Unlike adult practice, pathology in this paediatric cohort was very heterogenous.

Pathology

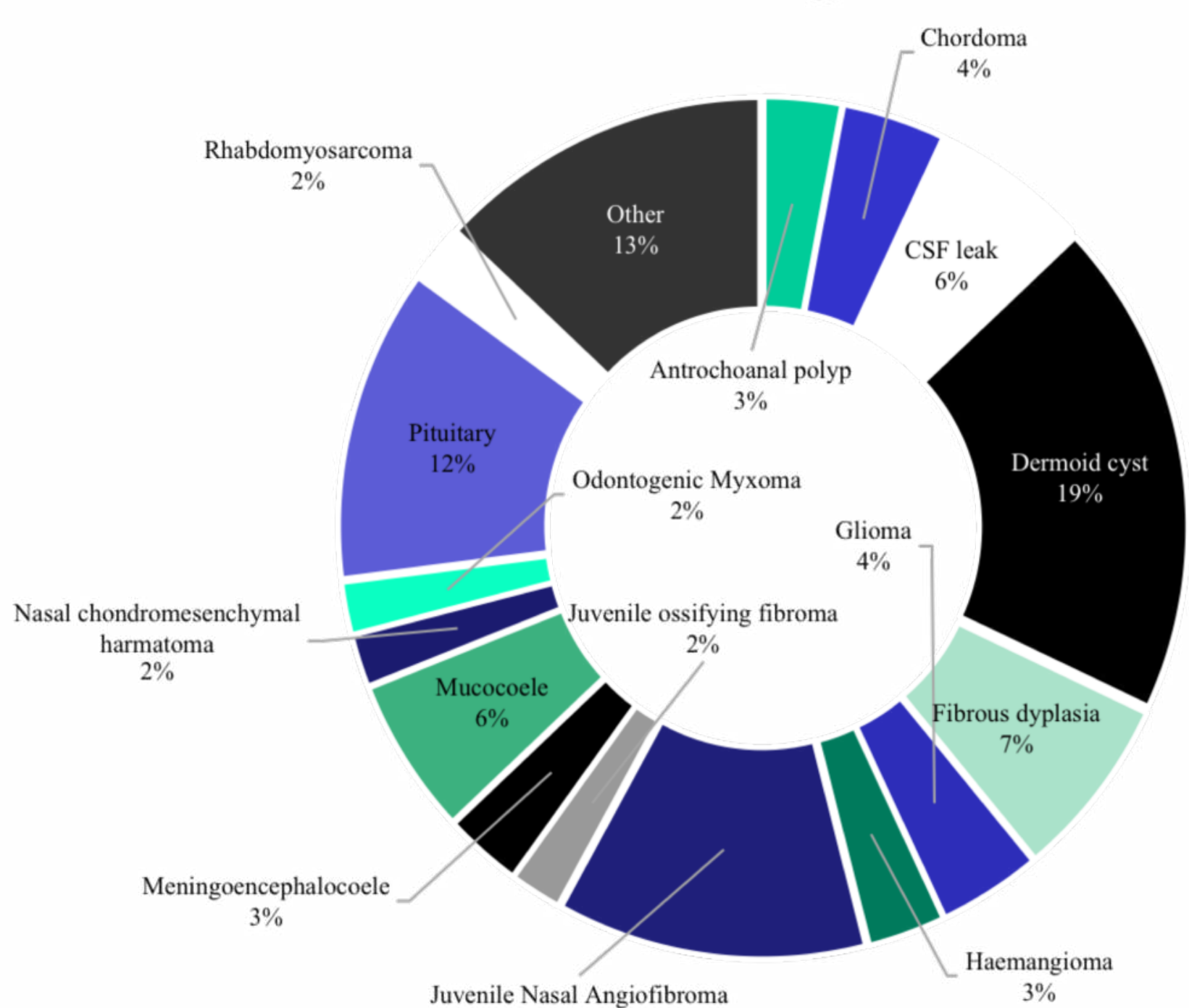

Early Complications $12 \%$

$3 \%$

- 10 temporary pituitary dysfunction ( $<12$ weeks)

- 5 post op CSF leaks

- 1 permanent unilateral visual loss

- 1 tension pneumocephalus

- 31 extensive nasal crusting

Late Complications

- 6 nasal saddle-deformities

- 2 mucocoele development

Facial symmetry \& growth does not appear to be significantly affected following endonasal surgery. A late saddle deformity is the main long-term problem despite minimal septal surgery.

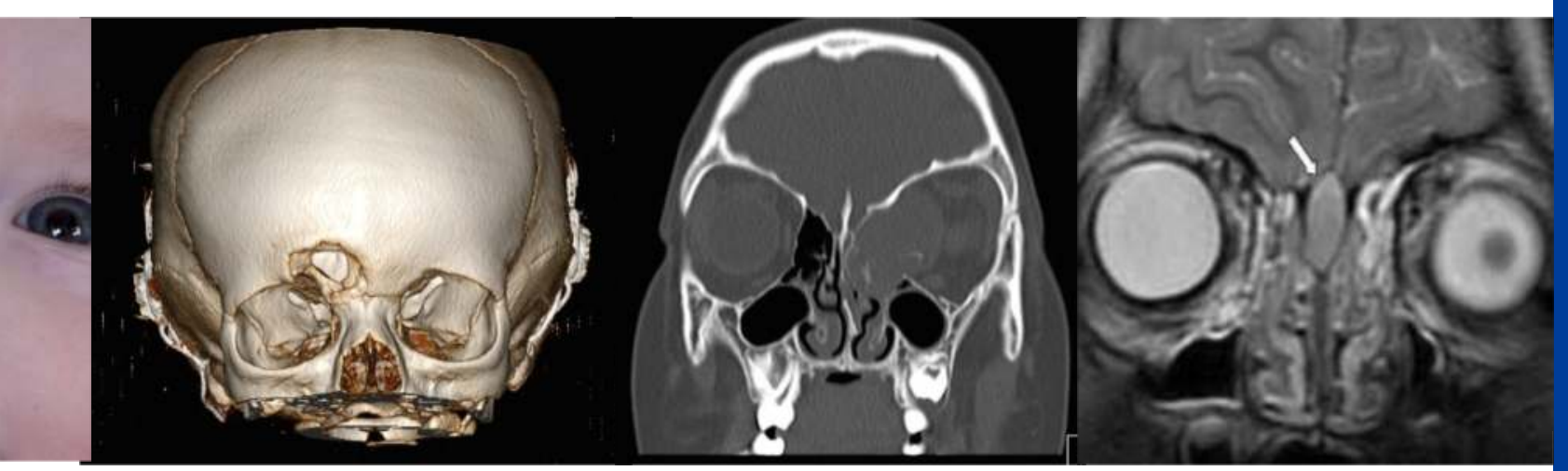

\section{Conclusion}

Endoscopic surgery is now well established in Birmingham Children's Hospital.

A dedicated multidisciplinary team is essential.

Early results show good outcomes and suggest minimal long-term effects on facial development. 